\title{
Extremal Isolated Horizon/CFT Correspondence
}

\author{
Xiao-Ning $\mathrm{Wu} *$ \\ Institute of Mathematics, Academy of Mathematics and System Science, \\ The Chinese Academy of Sciences, Beijing 100190, China and \\ Hua Loo-Keng Key Laboratory of Mathematics, \\ Chinese Academy of Sciences, Beijing 100190, China \\ Yu Tiant \\ College of Physical Sciences, \\ Graduate University of Chinese Academy of Sciences \\ Beijing 100049, China and \\ Kavli Institute for Theoretical Physics China, CAS \\ Beijing 100190, China
}

(Dated: October 28, 2018)

\begin{abstract}
The near-horizon limit of the extremal (weakly) isolated horizon is obtained under the Bondilike coordinates. For the vacuum case, explicit coordinate transformation relating the near-horizon metric under the Bondi-like coordinates and the standard Poincaré-type or global near-horizon metric of the extremal Kerr black hole is found, which shows that the two geometries are the same. Combined with the known thermodynamics of the (weakly) isolated horizon, it is argued that the Kerr/CFT correspondence can be generalized to the case of a large class of non-stationary extremal black holes.
\end{abstract}

*Electronic address: wuxn@amss.ac.cn

${ }^{\dagger}$ Electronic address: ytian@gucas.ac.cn; Tel: (86)10-82792321 


\section{INTRODUCTION}

The microscopic origin of the Bekenstein-Hawking entropy of various black holes is always an interesting problem in theoretical physics. As should eventually be given by microstate counting in quantum statistics, this entropy provides important information from the quantum theory of gravity. In fact, since the first successful microstate counting of the five-dimensional extremal black holes by Strominger and Vafa [1] and the four-dimensional extremal black holes by Maldacena and Strominger [2] in the context of superstring theory, it has been shown by Strominger [3] that for the BTZ black hole the correct microstate counting can be done in a dual two-dimensional CFT without appealing to supersymmetry or string theory, based on the work by Brown and Henneaux [4].

A recent, essential progress on this subject is the microstate counting of the fourdimensional extremal Kerr black hole via a dual chiral two-dimensional CFT living on the boundary of the near-horizon extremal Kerr (NHEK) geometry [5]. This work has been extended to black holes in diverse dimensions and/or with $U(1)$ gauge symmetries in the framework of Einstein-Maxwell(-scalar), supergravity or superstring theory [6, 7, 8, 9, 10, 11, 12, 13, 14, 15, 16, 17, 18]. Some related developments can be found

in [19, 20]. Furthermore, classification of near-horizon geometries of stationary extremal black holes, including those with supersymmetries and/or in higher dimensions also has been carefully studied, with some powerful theorems proved [21, 22].

All the known examples, however, of the so-called Kerr/CFT correspondence are for stationary Kerr-type black holes, i.e. each of them study black holes in stationary and axial symmetric spacetime. An interesting question is whether such correspondence is right for more general black holes, especially for non-stationary black holes. In order to consider this problem, we need a general definition of the horizon. Weakly isolated horizon (WIH) is a nice choice [23]. A nice review on the theory of WIH can be found in [24]. WIH is a generalization of the Killing horizon of black hole. It not only covers almost all known Killing horizons, including Schwarzschild and Kerr horizons, but also contains many non-stationary cases [25]. On the other hand, the thermodynamics of WIH has been studied extensively, with almost all elegant results as in the stationary case obtained [26]. It has also been shown that Hawking radiation exists near WIH [27]. It is then natural and interesting to provide a microscopic interpretation for the entropy of WIH, possibly by seeking the generalization 
of the Kerr/CFT correspondence to the (weakly) isolated horizon case, which is the main purpose of our paper. In fact, we will show that this is really possible for the vacuum, extremal isolated horizon.

This paper is organized as follows. In Sec. III, we introduce the general definition of the (weakly) isolated horizon and work out its near-horizon geometry in the extremal case. In Sec. III, we take its near-horizon limit. For the vacuum, extremal isolated horizon, we obtain the explicit form of the near-horizon limit and find the explicit coordinate transformation to the standard Poincaré-type or global near-horizon metric of the extremal Kerr black hole, which combined with the known thermodynamics of this kind of isolated horizon validates the microscopic interpretation for the corresponding entropy via a dual chiral twodimensional CFT. Finally, we conclude and make some discussions in Sec. IV.

\section{EXTREMAL (WEAKLY) ISOLATED HORIZON AND ITS NEAR-HORIZON GEOMETRY}

Based on works by A. Ashtekar and his colleagues [24], the weakly isolated horizon and isolated horizon are defined as

Definition 1 (weakly isolated horizon)

Let $(M, g)$ be a space-time. $\mathcal{H}$ is a 3-dim null hyper-surface in $M$ and $l^{a}$ is the tangent vector filed of the generator of $\mathcal{H} . \mathcal{H}$ is said to be a weakly isolated horizon (WIH), if

1. $\mathcal{H}$ has the topology of $S^{2} \times \Re$,

2. The expansion of the null generator of $\mathcal{H}$ is zero, i.e. $\Theta_{l}=0$ on $\mathcal{H}$,

3. $T_{a b} v^{b}$ is future causal for any future causal vector $v^{a}$ and the Einstein equation holds in a neighborhood of $\mathcal{H}$,

4. $\left[\mathcal{L}_{l}, D_{a}\right] l^{a}=0$ on $\mathcal{H}$, where $D_{a}$ is the induced covariant derivative on $\mathcal{H}$.

Definition 2 (isolated horizon)

Let $(M, g)$ be a space-time. $\mathcal{H}$ is a 3-dim null hyper-surface in $M$ and $l^{a}$ is the tangent vector filed of the generator of $\mathcal{H} . \mathcal{H}$ is said to be an isolated horizon (IH), if

1. $\mathcal{H}$ has the topology of $S^{2} \times \Re$, 
2. The expansion of the null generator of $\mathcal{H}$ is zero, i.e. $\Theta_{l}=0$ on $\mathcal{H}$,

3. $T_{a b} v^{b}$ is future causal for any future causal vector $v^{a}$ and the Einstein equation holds in a neighborhood of $\mathcal{H}$,

4. $\left[\mathcal{L}_{l}, D_{a}\right]=0$ on $\mathcal{H}$, where $D_{a}$ is the induced covariant derivative on $\mathcal{H}$.

So the fourth condition for the latter is stronger.

We need to choose coordinates and tetrad to describe their near-horizon geometry. For the WIH, a convenient choice is the Bondi-like coordinates. The coordinates are constructed as follows: The tangent vector of null generator of $\mathcal{H}$ is $l^{a}$; Another real null vector field is $n^{a}$; The foliation of $\mathcal{H}$ gives us the natural coordinates $(\theta, \phi)$; Using a parameter $u$ of $l^{a}$ and Lie dragging $(\theta, \phi)$ along each generator of $\mathcal{H}$, we have coordinates $(u, \theta, \phi)$ on $\mathcal{H}$; Choosing the affine parameter $r$ of $n^{a}$ as the forth coordinate, we obtain the Bondi-like coordinates $(u, r, \theta, \phi)$ near the horizon ${ }^{1}$. In these coordinates, we choose the null tetrad as

$$
\begin{aligned}
l^{a} & =\frac{\partial}{\partial u}+U \frac{\partial}{\partial r}+X \frac{\partial}{\partial \zeta}+\bar{X} \frac{\partial}{\partial \bar{\zeta}} \\
n^{a} & =\frac{\partial}{\partial r} \\
m^{a} & =\omega \frac{\partial}{\partial r}+\xi^{3} \frac{\partial}{\partial \zeta}+\xi^{4} \frac{\partial}{\partial \bar{\zeta}} \\
\bar{m}^{a} & =\bar{\omega} \frac{\partial}{\partial r}+\bar{\xi}^{3} \frac{\partial}{\partial \bar{\zeta}}+\bar{\xi}^{4} \frac{\partial}{\partial \zeta}
\end{aligned}
$$

where $U=X=\omega=0$ on $\mathcal{H}$ (following the notation in [24], equalities restricted to $\mathcal{H}$ will be denoted by "ᄉ्=" hereafter), and $(\zeta, \bar{\zeta})$ are complex coordinates on the section of $\mathcal{H}$ (not necessarily $e^{ \pm i \phi} \cot \frac{\theta}{2}$ ). In words of the Newman-Penrose formulism, we also require the above tetrad satisfy the following gauge:

$$
\begin{aligned}
& \nu=\gamma=\tau=\alpha+\bar{\beta}-\pi=\mu-\bar{\mu}=0, \\
& \varepsilon-\bar{\varepsilon} \hat{=} \kappa \hat{=} 0
\end{aligned}
$$

which means these tetrad vectors are parallelly transported along $n^{a}$ in space-time. Furthermore, the forth requirement in the definition of WIH implies there exists a one form $\omega_{a}$ on $\mathcal{H}$ such that $D_{a} l^{b} \hat{=} \omega_{a} l^{b}$ and $\mathcal{L}_{l} \omega_{a} \hat{=} 0$. In terms of the Newman-Penrose formulism,

\footnotetext{
${ }^{1}$ The Bondi-like coordinates here are exactly the so-called Gaussian null coordinates in [21, 22].
} 
$\omega_{a}$ can be expressed as $\omega_{a}=-(\varepsilon+\bar{\varepsilon}) n_{a}+(\alpha+\bar{\beta}) \bar{m}_{a}+(\bar{\alpha}+\beta) m_{a}$. The above equation means $(\varepsilon+\bar{\varepsilon})$ is constant on $\mathcal{H}$. Based on the work of Astekar et. al. [23, 24], we know the surface gravity of WIH is $\kappa_{t}=\omega_{a} l^{a}$ [24], so the extremal condition tells us that $\varepsilon+\bar{\varepsilon} \hat{=} 0$. In general, the inverse metric has the following form:

$$
\left(g^{\mu \nu}\right)=\left(\begin{array}{cccc}
0 & -1 & 0 & 0 \\
-1 & 2\left(|\omega|^{2}-U\right) & \bar{\omega} \xi^{3}+\omega \bar{\xi}^{4}-X & \bar{\omega} \xi^{4}+\omega \bar{\xi}^{3}-\bar{X} \\
0 & \bar{\omega} \xi^{3}+\omega \bar{\xi}^{4}-X & 2 \xi^{3} \bar{\xi}^{4} & \left|\xi^{3}\right|^{2}+\left|\xi^{4}\right|^{2} \\
0 & \bar{\omega} \xi^{4}+\omega \bar{\xi}^{3}-\bar{X} & \left|\xi^{3}\right|^{2}+\left|\xi^{4}\right|^{2} & 2 \bar{\xi}^{3} \xi^{4}
\end{array}\right) .
$$

It is easy to see that $\sqrt{-g}=\sqrt{h}$ if we denote $\left(h_{A B}\right):=\left(m^{A} \bar{m}^{B}+\bar{m}^{A} m^{B}\right)^{-1}$ with $A, B=\theta, \phi$. Obviously, $h_{A B}$ is the induced metric on the section of WIH.

In order to control the geometry in the neighborhood of the horizon, we consider the commutative relations of tetrad (the first Cartan structure equation) [28]. The commutative relation of $l^{a}$ and $n^{a}$ is

$$
[n, l]=(\gamma+\bar{\gamma}) l+(\varepsilon+\bar{\varepsilon}) n-(\bar{\tau}+\pi) m-(\tau+\bar{\pi}) \bar{m}
$$

Using the Bondi gauge (2), we get

$$
[n, l]=(\varepsilon+\bar{\varepsilon}) n-\pi m-\bar{\pi} \bar{m} .
$$

The $\partial_{r}$ component of above equation is

$$
\frac{\partial U}{\partial r}=(\varepsilon+\bar{\varepsilon})-\bar{\pi} \bar{\omega}-\pi \omega
$$

So we know $\frac{\partial U}{\partial r} \hat{=}(\varepsilon+\bar{\varepsilon})$. The extremal condition tells us that $\partial_{r} U \hat{=} 0$. The $\partial_{\zeta}$ component of (5) is

$$
\partial_{r} X=-\pi \xi^{3}-\bar{\pi} \bar{\xi}^{4}
$$

For non-rotational case, $\pi \hat{=} 0$, so $\partial_{r} X \hat{=} 0$. In order to get $\left.\partial_{r} \omega\right|_{\mathcal{H}}$, we need to consider the commutative relation of $n^{a}$ and $m^{a}$ :

$$
[n, m]=\bar{\nu} l-(\tau-\bar{\alpha}-\beta) n-(\mu-\gamma+\bar{\gamma}) m-\bar{\lambda} \bar{m}
$$

Under the Bondi gauge, its $\partial_{r}, \partial_{\zeta}$ and $\partial_{\bar{\zeta}}$ components are

$$
\begin{aligned}
\partial_{r} \omega & =\bar{\pi}-\mu \omega-\bar{\lambda} \bar{\omega} \Rightarrow \partial_{r} \omega \hat{=} \bar{\pi} . \\
\partial_{r} \xi^{3} & =-\mu \xi^{3}-\bar{\lambda} \bar{\xi}^{4} \\
\partial_{r} \xi^{4} & =-\mu \xi^{4}-\bar{\lambda} \bar{\xi}^{3} .
\end{aligned}
$$


The value of $\xi^{3}$ and $\xi^{4}$ are always regular (here regular means finite and non-zero).

It turns out that, in order to consider the near-horizon limit, we need the value of $\partial_{r}^{2} U$ on horizon. From (6), it is easy to get

$$
\partial_{r}^{2} U=\left(\partial_{r} \varepsilon+\partial_{r} \bar{\varepsilon}\right)-\left(\partial_{r} \bar{\pi}\right) \bar{\omega}-\bar{\pi} \partial_{r} \bar{\omega}-\partial_{r} \pi \omega-\pi \partial_{r} \omega
$$

On horizon,

$$
\begin{aligned}
\partial_{r}^{2} U & \hat{=}\left(\partial_{r} \varepsilon+\partial_{r} \bar{\varepsilon}\right)-\bar{\pi} \partial_{r} \bar{\omega}-\pi \partial_{r} \omega \\
& \hat{=}\left(\partial_{r} \varepsilon+\partial_{r} \bar{\varepsilon}\right)-2|\pi|^{2} .
\end{aligned}
$$

So we need the value of $\partial_{r} \varepsilon$ on horizon. From N-P equations (the second Cartan structure equation) [28],

$$
D \gamma-D^{\prime} \varepsilon=(\tau+\bar{\pi}) \alpha+(\bar{\tau}+\pi) \beta-(\varepsilon+\bar{\varepsilon}) \gamma-(\gamma+\bar{\gamma}) \varepsilon+\tau \pi-\nu \kappa+\Psi_{2}-\frac{R}{24}+\Phi_{11} .
$$

Under the Bondi gauge, it becomes

$$
-\partial_{r} \varepsilon=\bar{\pi} \alpha+\pi \beta+\Psi_{2}-\frac{R}{24}+\Phi_{11}
$$

So

$$
\begin{aligned}
-\partial_{r} \varepsilon-\partial_{r} \bar{\varepsilon} & =\left(\bar{\pi} \alpha+\pi \beta+\Psi_{2}-\frac{R}{24}+\Phi_{11}\right)+\left(\pi \bar{\alpha}+\bar{\pi} \bar{\beta}+\bar{\Psi}_{2}-\frac{R}{24}+\Phi_{11}\right) \\
& =2|\pi|^{2}+2 \operatorname{Re} \Psi_{2}-\frac{R}{12}+2 \Phi_{11} .
\end{aligned}
$$

Now we consider the $u$-dependence of the near-horizon metric. On WIH, N-P equations tell us

$$
\begin{aligned}
& D \alpha-\bar{\delta} \varepsilon=(\rho+\bar{\varepsilon}-\varepsilon) \alpha+\beta \bar{\sigma}-\bar{\beta} \varepsilon-\kappa \lambda-\bar{\kappa} \gamma+(\varepsilon+\rho) \pi+\Phi_{10}, \\
& D \beta-\delta \varepsilon=(\alpha+\pi) \sigma+(\bar{\rho}-\bar{\varepsilon}) \beta-(\mu+\gamma) \kappa-(\bar{\alpha}-\bar{\pi}) \varepsilon+\Psi_{1} .
\end{aligned}
$$

The energy condition of WIH tells us $\Phi_{10} \hat{=} 0$, and the Raychaudhuri equation implies

$$
\rho \hat{=} 0, \quad \sigma \hat{=} 0, \quad \Psi_{0} \hat{=} 0, \quad \Psi_{1} \hat{=} 0
$$

The Bondi gauge and WIH condition then implies

$$
\partial_{u} \pi \hat{=} 0, \quad \partial_{u}\left(\Psi_{2}-\frac{R}{24}\right) \hat{=} 0
$$


WIH condition also gives

$$
\partial_{u} \xi^{3} \hat{=} 0, \quad \partial_{u} \xi^{4} \hat{=} 0
$$

We further impose the stability condition $\mathcal{L}_{l} \Phi_{11} \hat{=} 0$, which is automatically satisfied in the vacuum case.

To sum up, we get for WIH

$$
\begin{aligned}
U & =-\left(2|\pi|^{2}+\operatorname{Re}\left(\Psi_{2}\right)-\frac{R}{24}+\Phi_{11}\right) r^{2}+O\left(r^{3}\right), \\
\omega & =\bar{\pi} r+O\left(r^{2}\right), \\
X & =-\left(\pi \xi^{3}+\bar{\pi} \bar{\xi}^{4}\right) r+O\left(r^{2}\right) .
\end{aligned}
$$

For the vacuum case, it becomes

$$
\begin{aligned}
U & =-\left(2|\pi|^{2}+\operatorname{Re}\left(\Psi_{2}\right)\right) r^{2}+O\left(r^{3}\right), \\
\omega & =\bar{\pi} r+O\left(r^{2}\right), \\
X & =-\left(\pi \xi^{3}+\bar{\pi} \bar{\xi}^{4}\right) r+O\left(r^{2}\right) .
\end{aligned}
$$

The inverse metric has the following form

$$
\left(g^{\mu \nu}\right)=\left(\begin{array}{cccc}
0 & -1 & 0 & 0 \\
-1 & f_{1} r^{2}+O\left(r^{3}\right) & f_{2} r+O\left(r^{2}\right) & \bar{f}_{2} r+O\left(r^{2}\right) \\
0 & f_{2} r+O\left(r^{2}\right) & 2 \xi^{3} \bar{\xi}^{4} & \left|\xi^{3}\right|^{2}+\left|\xi^{4}\right|^{2} \\
0 & \bar{f}_{2} r+O\left(r^{2}\right) & \left|\xi^{3}\right|^{2}+\left|\xi^{4}\right|^{2} & 2 \bar{\xi}^{3} \xi^{4}
\end{array}\right)
$$

where

$$
f_{1}=6|\pi|^{2}+2 \operatorname{Re}\left(\Psi_{2}\right)-\frac{R}{12}+2 \Phi_{11}, \quad f_{2}=2\left(\pi \xi^{3}+\bar{\pi} \bar{\xi}^{4}\right)
$$

are functions of $\theta$ only. So the metric should be

$$
\left(g_{\mu \nu}\right)=\left(\begin{array}{ccc}
\left(h_{A B} f_{2}^{A} f_{2}^{B}-f_{1}\right) r^{2}+O\left(r^{3}\right) & -1 & h_{A B} f_{2}^{B} r+O\left(r^{2}\right) \\
-1 & 0 & \overrightarrow{0} \\
h_{A B} f_{2}^{A} r+O\left(r^{2}\right) & \overrightarrow{0} & h_{A B}
\end{array}\right), \quad A, B=\theta, \phi
$$

In the above calculation, because $l^{a}$ and $n^{a}$ are all chosen as future pointing, the outside black hole region corresponds to the region $r<0$. This is inconvenient for later use, so we 
introduce a simple coordinate reflection $r \rightarrow-r$ and re-express the above metric in the new coordinates as

$$
\left(g_{\mu \nu}\right)=\left(\begin{array}{ccc}
\left(h_{A B} f_{2}^{A} f_{2}^{B}-f_{1}\right) r^{2}+O\left(r^{3}\right) & -1 & -h_{A B} f_{2}^{B} r+O\left(r^{2}\right) \\
-1 & 0 & \overrightarrow{0} \\
-h_{A B} f_{2}^{A} r+O\left(r^{2}\right) & \overrightarrow{0} & h_{A B}
\end{array}\right), \quad A, B=\theta, \phi .
$$

\section{THE NEAR-HORIZON LIMIT OF EXTREMAL ISOLATED HORIZON AND ITS CFT DUAL}

Under the coordinate re-scaling

$$
u=\frac{\tilde{u}}{\lambda}, \quad r=\lambda \tilde{r}
$$

and taking the near-horizon limit $\lambda \rightarrow 0$ [5, 21, 29], the above metric becomes (after omitting all the tildes in the expression)

$$
d s^{2}=-f_{1} r^{2} d u^{2}-2 d u d r+\hat{h}_{A B}\left(d \zeta^{A}-f_{2}^{A} r d u\right)\left(d \zeta^{B}-f_{2}^{B} r d u\right)
$$

with $\hat{h}_{A B}=\left.h_{A B}\right|_{\mathcal{H}}$ now functions of $\theta$ only. This kind of near-horizon metrics have been obtained in [21, 22] and other papers, but here we achieve this directly from the viewpoint of extremal isolated horizon, without assuming any symmetry of the whole space-time.

The functions $f_{1}, f_{2}$ and $\hat{h}_{A B}$ satisfy certain geometric and dynamic constraints (while having some further gauge freedom), namely

$$
R_{m \bar{m}}=0, \quad R_{m m}=0 .
$$

These equations can be re-expressed in terms of the inner data $\left(\omega_{a}, \hat{h}_{a b}\right)$ of the horizon as

$$
\begin{aligned}
\widehat{\operatorname{div}} \hat{\omega}+\hat{\omega}^{2}-K & =0, \\
\nabla_{\bar{m}} \pi+2 \hat{\Gamma} \pi+\pi^{2} & =0,
\end{aligned}
$$

where "^" means projecting on the section $S^{2}$ of the horizon, $K$ is the Gaussian curvature of $S^{2}$, and $\hat{\Gamma}$ the component of connection on $S^{2}$. These equations have not yet been solved for the WIH and generic IH [24]. However, for the axi-symmetric, electrovac case of extremal IH, J. Lewandowski and T. Pawlowski have obtained the unique solution for these functions under certain stability condition for the electromagnetic field [30], i.e. the functions $f_{1}, f_{2}$ 
and $\hat{h}_{A B}$ (which are intrinsic data on horizon) are unique for the axi-symmetric, electrovac case.

Concerning the vacuum case, in which the stability condition for the electromagnetic field is automatically satisfied, the solution of (29) is [30]

$$
\begin{aligned}
\hat{m}^{a} & =\frac{1}{2}\left(P^{-1} \partial_{x}+i P \partial_{\phi}\right), \quad x=\cos \theta, \\
\hat{\omega}_{a} & =\hat{\star} d V+d \ln B
\end{aligned}
$$

with (taking the horizon area $A=8 \pi$ throughout our calculation)

$$
P^{2}=\frac{1+\cos ^{2} \theta}{2 \sin ^{2} \theta}, \quad V=-\arctan \cos \theta, \quad B=\left(1+\cos ^{2} \theta\right)^{1 / 2}
$$

and "रै" the Hodge star defined by the 2-metric $\hat{h}$. In fact, we have

$$
\hat{h}^{a b}=\hat{m}^{a} \hat{\bar{m}}^{b}+\hat{\bar{m}}^{a} \hat{m}^{b}=\frac{1}{2}\left(P^{-2} \partial_{x}^{2}+i P^{2} \partial_{\phi}^{2}\right),
$$

SO

$$
\hat{h}_{a b}=2\left(P^{2} d x^{2}+P^{-2} d \phi^{2}\right)=2\left(\frac{1+\cos ^{2} \theta}{2} d \theta^{2}+\frac{2 \sin ^{2} \theta}{1+\cos ^{2} \theta} d \phi^{2}\right) .
$$

Thus we obtain

$$
\hat{\omega}_{a}=-\frac{2 \sin ^{2} \theta}{\left(1+\cos ^{2} \theta\right)^{2}} d \phi-\frac{\cos \theta \sin \theta}{1+\cos ^{2} \theta} d \theta
$$

and

$$
\pi=-i \nabla_{\bar{m}} V+\nabla_{\bar{m}} \ln B=i \frac{1}{2 P} \frac{1}{1+\cos ^{2} \theta}+\frac{1}{2 P} \frac{\cos \theta}{1+\cos ^{2} \theta} .
$$

For vacuum IH we have $\operatorname{Re}\left(\Psi_{2}\right)=-K / 2$ with $K$ the Gaussian curvature of the 2-metric $\hat{h}$, which can be computed from (34) as

$$
K=\frac{2-6 \cos ^{2} \theta}{\left(1+\cos ^{2} \theta\right)^{3}}
$$

Noting in the vacuum case $R=0=\Phi_{11}$ in (23), we have

$$
\begin{array}{r}
f_{1}=6|\pi|^{2}+2 \operatorname{Re} \Psi_{2}=\frac{1+6 \cos ^{2} \theta-3 \cos ^{4} \theta}{\left(1+\cos ^{2} \theta\right)^{3}}, \\
\hat{h}_{A B} f_{2}^{A} d \zeta^{B}=2 \hat{\omega}_{a}=-\frac{4 \sin ^{2} \theta}{\left(1+\cos ^{2} \theta\right)^{2}} d \phi-\frac{2 \cos \theta \sin \theta}{1+\cos ^{2} \theta} d \theta,
\end{array}
$$

so, eventually, the near-horizon limit of vacuum, extremal IH is

$$
\begin{aligned}
d s^{2}= & -\frac{1+6 \cos ^{2} \theta-3 \cos ^{4} \theta}{\left(1+\cos ^{2} \theta\right)^{3}} r^{2} d u^{2}-2 d u d r+\left(1+\cos ^{2} \theta\right)\left(d \theta+\frac{2 r \cos \theta \sin \theta}{\left(1+\cos ^{2} \theta\right)^{2}} d u\right)^{2} \\
& +\frac{4 \sin ^{2} \theta}{1+\cos ^{2} \theta}\left(d \phi+\frac{r d u}{1+\cos ^{2} \theta}\right)^{2} .
\end{aligned}
$$


This can be shown to be the near-horizon limit of the extremal Kerr solution. In fact, under coordinate transformation

$$
\begin{aligned}
& u=t+\frac{1}{\rho}, \\
& r=\frac{1+\cos ^{2} \theta}{2} \rho, \\
& \phi=\varphi+\frac{1}{2} \ln \rho,
\end{aligned}
$$

the above metric becomes

$$
d s^{2}=\left(1+\cos ^{2} \theta\right)\left(-\frac{\rho^{2}}{4} d t^{2}+\frac{d \rho^{2}}{\rho^{2}}+d \theta^{2}\right)+\frac{4 \sin ^{2} \theta}{1+\cos ^{2} \theta}\left(d \varphi+\frac{\rho}{2} d t\right)^{2},
$$

which coincides with the near-horizon limit of the extremal Kerr solution in [29] with the ADM mass $M=1$ consistent with $A=8 \pi$ in our calculation. Restoring arbitrary $M$ and taking some trivial coordinate transformations, we obtain exactly the same Poincaré-type or global metrics of NHEK geometry as those in [5].

Now, according to [5], this NHEK geometry has an asymptotic symmetry group (under certain boundary conditions) containing diffeomorphisms generated by

$$
\xi_{\epsilon}=\epsilon(\varphi) \partial_{\varphi}-\rho \epsilon^{\prime}(\varphi) \partial_{\rho}
$$

which form a Virasoro algebra without central charge under Lie brackets. The conserved charges associated with these diffeomorphism generators [31] then form a Virasoro algebra, under Dirac brackets, with a central charge

$$
c_{L}=\frac{12 J}{\hbar}
$$

with $J=M^{2} / G$ the angular momentum of the extremal Kerr solution.

On the other hand, thermodynamics of general WIH has been extensively studied in the literature [26]. Ashtekar and Krishnan give a canonical way to choose the time direction $\partial_{\hat{t}}$ for general WIH, in order to get meaningful thermodynamics of the WIH, which actually defines the canonical coordinates $\hat{t}$ and $\hat{\phi}$ coinciding with the Boyer-Lindquist coordinates in the Kerr case [24]. Based on their results, for axial symmetric WIH, the surface gravity $\kappa_{\hat{t}}$, horizon angular velocity $\Omega_{\hat{t}}$ and horizon mass $M_{\hat{t}}$ associated with the canonical time also coincide with the corresponding quantities in the Kerr case. It has been shown that 
Hawking radiation exists near general WIH [27]. The temperature of this thermal radiation is $T=\frac{\kappa_{\hat{t}}}{2 \pi}$, so the Boltzmann factor observed by a canonical observer should be

$$
\exp \left(-\frac{E-m \Omega_{\hat{t}}}{T}\right)
$$

where $E$ is the particle energy and $m$ the particle angular momentum associated with the canonical observer. We also introduce coordinates $t, \phi$ as

$$
t=\frac{\lambda \hat{t}}{2 M_{\hat{t}}}, \quad \phi=\hat{\phi}-\Omega_{\hat{t}} \hat{t},
$$

which coincide, in the extremal case, with the coordinates $t, \phi$ in [5]. Then the particle energy and angular momentum associated with the new observer are

$$
n_{R}=\left(2 M_{\hat{t}} E-m\right), \quad n_{L}=m
$$

so we can reexpress the Boltzmann factor as

$$
\exp \left(-\frac{E-m \Omega_{\hat{t}}}{T}\right)=\exp \left(-\frac{n_{L}}{T_{L}}-\frac{n_{R}}{T_{R}}\right) .
$$

Under the extreme limit $J \rightarrow M^{2} / G$, it is easy to get

$$
T_{L}=\frac{1}{2 \pi}, \quad T_{R}=0
$$

This means that the left modes are then thermally distributed with the temperature $\frac{1}{2 \pi}$.

Upon applying the Cardy formula, the microscopic entropy for the CFT dual to the vacuum, extremal IH is

$$
S_{\mathrm{CFT}}=\frac{\pi^{2}}{3} c_{L} T_{L}=\frac{2 \pi J}{\hbar}
$$

which is the same as the macroscopic entropy $S_{\mathrm{IH}}$ of the vacuum, extremal IH [24].

\section{CONCLUDING REMARKS}

In this paper, we show that the entropy of the vacuum, extremal isolated horizon may have microscopic origin, by working out its near-horizon limit, finding the explicit coordinate transformation to the standard Poincaré-type or global near-horizon metric of the extremal Kerr black hole, and using the original Kerr/CFT argument. This supports the generalization of the Kerr/CFT correspondence to the extremal isolated horizon/CFT correspondence. Based on our discussion, the near horizon geometry of IH is fixed by the inner data of the 
horizon, i.e. $\left(h_{a b}, \omega_{a}\right)$. This may tell us that the origin of black hole entropy is only related with the inner geometry of the horizon and does nothing with how we embed it into the space-time, at least in the framework of Einstein gravity.

The near-horizon limit that we obtain is for the general weakly isolated horizon, but only for the special case of the vacuum, extremal isolated horizon exact solution of the intrinsic data on the horizon is known. It is expected that more general cases can be dealt with, when there is no explicit solution and more general boundary conditions for the asymptotic symmetry of the near-horizon geometry have to be found. This is left for future work.

\section{Acknowledgments}

We thank Dr. W. Song, Prof. C.-G. Huang and Prof. Y. Yang for helpful discussions. This work is partly supported by the National Natural Science Foundation of China (Grant Nos. 10605005, 10705048, 10605006 and 10731080) and the President Fund of GUCAS.

[1] A. Strominger and C. Vafa, Phys. Lett. B 379 (1996) 99 hep-th/9601029.

[2] J.M. Maldacena and A. Strominger, Phys. Rev. Lett. 77 (1996) 428 hep-th/9603060].

[3] A. Strominger, JHEP 9802 (1998) 009 [hep-th/9712251].

[4] J.D. Brown and M. Henneaux, Commun. Math. Phys. 104, 207 (1986).

[5] M. Guica, T. Hartman, W. Song and A. Strominger, The Kerr/CFT Correspondence, arXiv:0809.4266.

[6] K. Hotta, Y. Hyakutake, T. Kubota, T. Nishinaka and H. Tanida, The CFT-interpolating Black Hole in Three Dimensions, JHEP 0901 (2009) 010 [arXiv:0811.0910].

[7] H. Lu, J. Mei and C.N. Pope, Kerr-AdS/CFT Correspondence in Diverse Dimensions, arXiv:0811.2225.

[8] T. Azeyanagi, N. Ogawa and S. Terashima, Holographic Duals of Kaluza-Klein Black Holes, arXiv:0811.4177]; The Kerr/CFT Correspondence and String Theory, arXiv:0812.4883.

[9] T. Hartman, K. Murata, T. Nishioka and A. Strominger, CFT Duals for Extreme Black Holes, arXiv:0811.4393. 
[10] D.D.K. Chow, M. Cvetic, H. Lu and C.N. Pope, Extremal Black Hole/CFT Correspondence in (Gauged) Supergravities, arXiv:0812.2918].

[11] Y. Nakayama, Emerging AdS from Extremally Rotating NS5-branes, arXiv:0812.2234.

[12] H. Isono, T.-S. Tai and W.-Y. Wen, Kerr/CFT correspondence and five-dimensional BMPV black holes, arXiv:0812.4440.

[13] J.-J. Peng and S.-Q. Wu, Phys. Lett. B 673 (2009) 216-219 [arXiv:0901.0311].

[14] C.-M. Chen and J.E. Wang, Holographic Duals of Black Holes in Five-dimensional Minimal Supergravity, arXiv:0901.0538.

[15] A.M. Ghezelbash, Kerr/CFT Correspondence in Low Energy Limit of Heterotic String Theory, arXiv:0901.1670.

[16] H. Lu, J. Mei, C.N. Pope and J. Vazquez-Poritz, Phys. Lett. B 673 (2009) 77-82 arXiv:0901.1677.

[17] G. Compère, K. Murata and T. Nishioka, Central Charges in Extreme Black Hole/CFT Correspondence, arXiv:0902.1001.

[18] D. Astefanesei and Y.K. Srivastava, CFT Duals for Attractor Horizons, arXiv:0902.4033.

[19] C. Krishnan and S. Kuperstein, A Comment on Kerr-CFT and Wald Entropy, arXiv:0903.2169.

[20] M.R. Garousi and A. Ghodsi, The RN/CFT Correspondence, arXiv:0902.4387].

[21] H.S. Reall, Phys. Rev. D 68 (2003) 024024;

H.K. Kunduri, J. Lucietti and H.S. Reall, Class. Quant. Grav. 24 (2007) 4169.

[22] H.K. Kunduri and J. Lucietti, A classification of near-horizon geometries of extremal vacuum black holes, arXiv:0806.2051];

H.K. Kunduri and J. Lucietti, Class. Quant. Grav. 26 (2009) 055019.

[23] A. Ashtekar, C. Beetle and S. Fairhurst, Class. Quant. Grav. 16 (1999) L1.

A. Ashtekar, C. Beetle, O. Dreyer, S. Fairhurst, B. Krishnan, J. Lewandowski and J. Wisniewski, Phys. Rev. Lett. 85 (2000) 3564.

A. Ashtekar, C. Beelte and J. Lewandowski, Class. Quant. Grav. 19 (2002) 1195.

[24] A. Ashtekar and B. Krishnan, Living Rev. Rel. 7 (2004) 10.

[25] J. Lewandowski, Class. Quant. Grav. 17 (2000) L53-L59.

[26] A. Ashtekar, C. Beetle and S. Fairhurst, Class. Quant. Grav. 17 (2000) 253.

A. Ashtekar, S. Fairhurst and B. Krishnan, Phys. Rev. D 62 (2000) 104025. 
A. Ashtekar, C. Beetle and J. Lewandowski, Phys. Rev. D 64 (2001) 044016.

[27] X.-N. Wu and S.-J. Gao, Phys. Rev. D 75 (2007) 044027.

X.-N. Wu, C.-G. Huang and J.-R. Sun, Phys. Rev. D 77 (2008) 124023.

[28] D. Kramer, H. Stephani, E. Herlt and M. MacCallum, Exact Solutions of Einstein's Field Equations, Cambridge University Press, 1980.

[29] J.M. Bardeen and G.T. Horowitz, Phys. Rev. D 60 (1999) 104030 hep-th/9905099.

[30] J. Lewandowski and T. Pawlowski, Class. Quant. Grav. 20 (2003) 587-606.

[31] T. Regge and C. Teitelboim, Annals Phys. 88 (1974) 286.

V. Iyer and R.M. Wald, Phys. Rev. D 50 (1994) 846 gr-qc/9403028.

G. Barnich, F. Brandt and M. Henneaux, Commun. Math. Phys. 174 (1995) 57.

G. Barnich, F. Brandt and M. Henneaux, Phys. Rept. 338 (2000) 439 hep-th/0002245.

G. Barnich and F. Brandt, Nucl. Phys. B 633 (2002) 3 hep-th/0111246].

G. Compere and S. Detournay, JHEP 0703 (2007) 098. 